\title{
Search for neutrino bursts from collapsing stars with the MACRO detector
}

\section{MACRO Collaboration}

S. Ahlen ${ }^{\text {c }}$, M. Ambrosio ', R. Antolini ${ }^{\text {b }}$, G. Auriemma ${ }^{\mathrm{n}, 1}$, R. Baker ${ }^{\mathrm{k}}$, A. Baldini ${ }^{\mathrm{m}}$, G.C. Barbarino ', B.C. Barish ${ }^{\mathrm{d}}$, G. Battistoni ${ }^{\mathrm{f}}$, R. Bellotti ${ }^{\mathrm{a}}$, C. Bemporad ${ }^{\mathrm{m}}$, P. Bernardini ${ }^{\mathrm{j}}$, H. Bilokon ${ }^{\text {f }}$, V. Bisi ${ }^{\text {p}}$, C. Bloise ${ }^{\text {f }}$, C. Bower ${ }^{\mathrm{h}}$, S. Bussino ${ }^{\mathrm{n}}$, F. Cafagna a ${ }^{\text {a }}$ M. Calicchio a , P. Campana ${ }^{\text {f }}$, D. Campana ', M. Carboni ${ }^{\text {f }}$, S. Cecchini ${ }^{\text {b, }}$, F. Cei ${ }^{\mathrm{m}}$, V. Chiarella ${ }^{\mathrm{f}}$, C. Chiera ${ }^{\mathrm{n}}$, A. Cobis ${ }^{\mathrm{f}}, \mathrm{R}$. Cormack ${ }^{\mathrm{c}}$, A. Corona ${ }^{\mathrm{n}}, \mathrm{S}$. Coutu ${ }^{\mathrm{d}}$, G. DeCataldo a, C. DeMarzo a , M. De Vincenzi ${ }^{n}$, A. Di Credico ${ }^{i}$, E. Diehl ${ }^{\text {k }}$, H. Dekhissi ${ }^{b}$, O. Erriquez ${ }^{a}$, C. Favuzzi ${ }^{a}$, D. Ficenec ${ }^{\text {c, } 3}$, C. Forti ${ }^{\text {f }}$, L. Foti ${ }^{n}$, P. Fusco ${ }^{a}$, G. Giacomelli ${ }^{b}$, G. Giannini ${ }^{m, 4}$, N. Giglietto ${ }^{a}$, P. Giubellino ${ }^{p}$, M. Grassi ${ }^{m}$, P. Green r ${ }^{\text {, A. Grillo }}{ }^{\text {f }}$, F. Guarino ${ }^{\text {}, ~ C . ~ G u s t a v i n o ~}{ }^{\mathrm{g}}$, A. Habig ${ }^{\text {h }}$, R. Heinz ${ }^{\text {h }}$, J.T. Hong ${ }^{\text {d, }}$ E. Iarocci ${ }^{\text {f,5 }}$, E. Katsavounidis ${ }^{\text {d, }}$, E. Kearns ${ }^{\text {c }}$, S. Klein ${ }^{\text {c,6 }}$, S. Kyriazopoulou ${ }^{\text {d, }}$, E. Lamanna ${ }^{\text {n }}$, C. Lane ${ }^{\text {e }}$, C. Lee ${ }^{\text {k }}$, D. Levin ${ }^{\text {k }}$, P. Lipari ${ }^{n}$, G. Liu ${ }^{\text {d }}$, R. Liu ${ }^{\text {d, }}$ M.J. Longo ${ }^{k}$, G. Ludlam ${ }^{c}$, G. Mancarella ${ }^{j}$, G. Mandrioli ${ }^{b}$, A. Margiotta-Neri ${ }^{b}$, A. Marin ${ }^{\text {c }}$, A. Marini ${ }^{\text {f }}$, D. Martello ${ }^{\text {j}}$, G. Martellotti ${ }^{\text {n, A. Marzari Chiesa }}{ }^{\text {p }}$, M. Masera ${ }^{\text {p }}$, P. Matteuzzi ${ }^{\text {b }}$, D.G. Michael ${ }^{\text {d }}$, L. Miller ${ }^{\text {h}}$, P. Monacelli ${ }^{i}$, M. Monteno ${ }^{\text {P, }}$ S. Mufson ${ }^{\text {h}}$, J. Musser h, D. Nicoló ${ }^{\text {m}}$, S. Nutter ${ }^{\text {h,7 }}$, C. Okada ${ }^{\text {c }}$, G. Osteria ', O. Palamara ${ }^{j}$, S. Parlati ${ }^{\mathrm{g}}$, V. Patera ${ }^{\mathrm{f}}$, L. Patrizii ${ }^{b}$, R. Pazzi ${ }^{\mathrm{m}}$, C.W. Peck ${ }^{\mathrm{d}}$, J. Petrakis ${ }^{\mathrm{q}}$, S. Petrera ${ }^{j}$, N.D. Pignatano ${ }^{d}$, P. Pistilli ${ }^{j}$, F. Predieri ${ }^{b}$, L. Ramello ${ }^{p}$, J. Reynoldson ${ }^{g}$, F. Ronga ${ }^{f}$, G. Rosa ${ }^{n}$, C. Satriano ${ }^{\mathrm{n}, 1}$, L. Satta ${ }^{\mathrm{f}, 5}$, E. Scapparone ${ }^{\mathrm{b}}$, K. Scholberg ${ }^{\mathrm{d}}$, A. Sciubba ${ }^{\text {n,5 }}$, P. Serra Lugaresi ${ }^{b}$, M. Severi ${ }^{n}$, M. Sitta ${ }^{\text {p }}$, P. Spinelli ${ }^{a}$, M. Spinetti ${ }^{\text {f }}$, M. Spurio ${ }^{b}$, J. Steele ${ }^{d}$, R. Steinberg ${ }^{\text {c }}$, J.L. Stone ${ }^{c}$, L.R. Sulak ${ }^{c}$, A. Surdo ${ }^{j}$, G. Tarlé ${ }^{k}$, V. Togo ${ }^{b}$, V. Valente ${ }^{\mathrm{f}}$, G.R. Verdone ${ }^{\mathrm{n}, 1}$, C.W. Walter ${ }^{\mathrm{d}}, \mathrm{R}$. Webb ${ }^{\circ}$, W. Worstell ${ }^{\mathrm{c}}$

a Dipartimento di Fisica dell'Università di Bari and INFN, Bari, 70126, Italy

${ }^{\circ}$ Dipartimento di Fisica dell'Università di Bologna and INFN, Bologna, 40126, Italy

' Physics Department, Boston University, Boston, MA 02215, USA

'California Institute of Technology, Pasadena, CA 91125, USA

'Department of Physics, Drexel University, Philadelphia, PA 19104, USA

${ }^{f}$ Laboratori Nazionali di Frascati dell'INFN, Frascati (Roma), 00044, Italy

${ }^{g}$ Laboratori Nazionali del Gran Sasso dell INFN, Assergi (L'Aquila), 67010, Italy

${ }^{h}$ Departments of Physics and of Astronomy, Indiana University, Bloomington, IN 4740S, USA

'Dipartimento di Fisica dell'Università dell'Aquila and INFN, L'Aquila, 67100, Italy

'Dipartimento di Fisica dell'Università di Lecce and INFN, Lecce, 73100, Italy

${ }^{*}$ Department of Physics, University of Michigan, Ann Arbor, MI 48109, USA

'Dipartimento di Fisica dell'Università di Napoli and INFN, Napoli, 80125, Italy

" Dipartimento di Fisica dell Università di Pisa and INFN, Pisa 56010, Italy

${ }^{n}$ Dipartimento di Fisica dell'Università di Roma and INFN, Roma, 00185, Italy

- Physics Department, Texas A\&M University, College Station, TX 77843, USA

${ }^{\circ}$ Dipartimento di Fisica dell'Università di Torinto and INFN, Torino, 10125, Italy 


\footnotetext{
${ }^{9}$ Bartol Research Institute, University of Delaware, Newark, DE 19716, USA

'Sandia National Laboratory, Albuquerque, NM 87185, USA
}

Received 26 May 1992

\begin{abstract}
The MACRO detector has been used to perform a search for bursts of neutrinos from Galactic supernovæ from October 1989 to February 1992. We did not detect any burst compatible with a stellar core collapse during this interval. With an active mass of 45 tonnes of liquid scintillator, our search extended to over $60 \%$ of the stars in the Galaxy. Both the current and planned future capabilities of MACRO to detect supernova are described.
\end{abstract}

\section{Introduction}

The successful detection of neutrinos of stellar origin by underground experiments opened a new field of observational astronomy - low-energy neutrino astrophysics. Neutrinos, the first nonelectromagnetic probes used for astronomical investigation, can carry information directly from stellar interiors. Solar neutrinos provide informa* tion on stellar burning process, whereas supernova neutrinos can give new insight into the final evolutionary stages of very massive stars.

The $\bar{\nu}_{\mathrm{e}}$ burst from SN1987A was unambiguously detected by the KAMIOKANDE-II and IMB experiments [1] and possibly by the Mt. Blanc and Baksan detectors [2]. The large fiducial volumes of these water Čerenkov experiments [1] enabled the first non-optical observation of an extragalactic supernova. The number of neutrino events was small due to the large distance (50 kpc) to the Large Magellanic Cloud (LMC), but was sufficient to confirm the main predictions for

Correspondence to: Prof. M. Longo, Department of Physics, University of Michigan, Ann Arbor, MI 48109, USA.

I Also at: Università della Basilicata, Potenza, 85100, Italy.

${ }^{2}$ Also at: Istituto TESRE/CNR, Bologna, Italy.

${ }^{3}$ Now at: Physics Department, Washington University, St Louis, MO 63130, USA.

4 Also at: Università di Trieste and INFN, Trieste, 34100, Italy.

5 Also at: Dipartimento di Energetica, Università di Roma, Roma, 00185, Italy.

"Now at: Department of Physics, University of California, Santa Cruz, CA 95064, USA.

${ }^{7}$ Currently at: Laboratori Nazionali del Gran Sasso dell'INFN, 67010, Italy. type II supernovæ. It is reasonable to expect that a Galactic supernova occurring at a smaller distance $(\sim 8 \mathrm{kpc})$ would provide more detailed information bearing directly on both astrophysical models of stellar collapse and elementary particle physics.

MACRO [3] is a large liquid scintillator and streamer tube experiment which is sufficiently completed now to operate as a new Galactic supernova observatory. Liquid scintillator experiments have not reached the volumes of large water Čerenkov detectors, but nevertheless have interesting capabilities, including superior energy resolution and potentially lower energy thresholds.

MACRO is located in Hall B of the Gran Sasso Laboratory (120 km east of Rome) at a minimum depth of $3200 \mathrm{~m}$ water equivalent. At this depth, the flux of cosmic ray muons reaching the experiment is reduced by a factor greater than $10^{6}$ relative to sea level fluxes. The detector is composed of six similar supermodules (SMs) and when completed will have dimensions 72 $\mathrm{m} \times 12 \mathrm{~m} \times 9 \mathrm{~m}$. Its large area will make MACRO well suited for the study of high-energy downward-going muons produced by cosmic ray nuclei and $\gamma$-rays interacting at the top of the atmosphere, as well as upward-going muons originating in high-energy neutrino interactions in the rock below. MACRO will search for rare penetrating heavy particles and will reach a sensitivity to the magnetic monopole flux well beyond the Parker bound [4]. Finally, it will have a large liquid scintillator mass and thus will be an important low-energy neutrino observatory for the study of supernovæ (but not solar neutrinos because of the large background at very low energies). 
Presently the lower part of the 6 supermodules has been built and is scheduled to become operational by summer 1992. The sensitive mass is expected to reach 600 tonnes by the end of 1992 . A final design mass of 1000 tonnes could be reached in a future extension, still under study. The data presented and discussed in this paper were primarily collected by SM1 during approximately 2.5 years of operation. A few months of data were also collected by SM2 and SM3 and are included in the data sample presented.

This paper describes the capabilities of the MACRO detector for low-energy stellar collapse neutrino physics. This running period of about 2.5 years was used for the supernova search, as well as to evaluate experimental requirements, backgrounds, calibration procedures, etc., that will be needed for the full detector. The analysis of this initial search is presented, and enhancements which are being implemented are described.

\section{Supernova theory and neutrino burst produc- tion}

Before discussing the capabilities of the detector, we will describe the neutrino output of a type II supernova. The evolution of a massive star including its final gravitational collapse has been extensively simulated with theoretical models (for a review see ref. [5]). The detection of the SN1987A $\bar{v}_{\mathrm{e}}$ burst largely confirmed the theoretical expectations.

At the end of a series of thermonuclear burning stages, a central iron-nickel core supported by electron degeneracy pressure reaches a temperature of $T=10^{10} \mathrm{~K}$ and a density of $10^{10}$ $\mathrm{g} / \mathrm{cm}^{3}$. Under these conditions, electron capture $\mathrm{e}^{-}+\mathrm{p} \rightarrow \nu_{\mathrm{e}}+\mathrm{n}$ becomes the dominant reaction, and an initial burst of $v_{\mathrm{e}}$ lasting roughly $10 \mathrm{~ms}$ is produced. The average $v_{\mathrm{e}}$ energy is $10 \mathrm{MeV}$, and the total energy released is $10^{52}$ ergs. With no further nuclear fusion reactions occurring in the

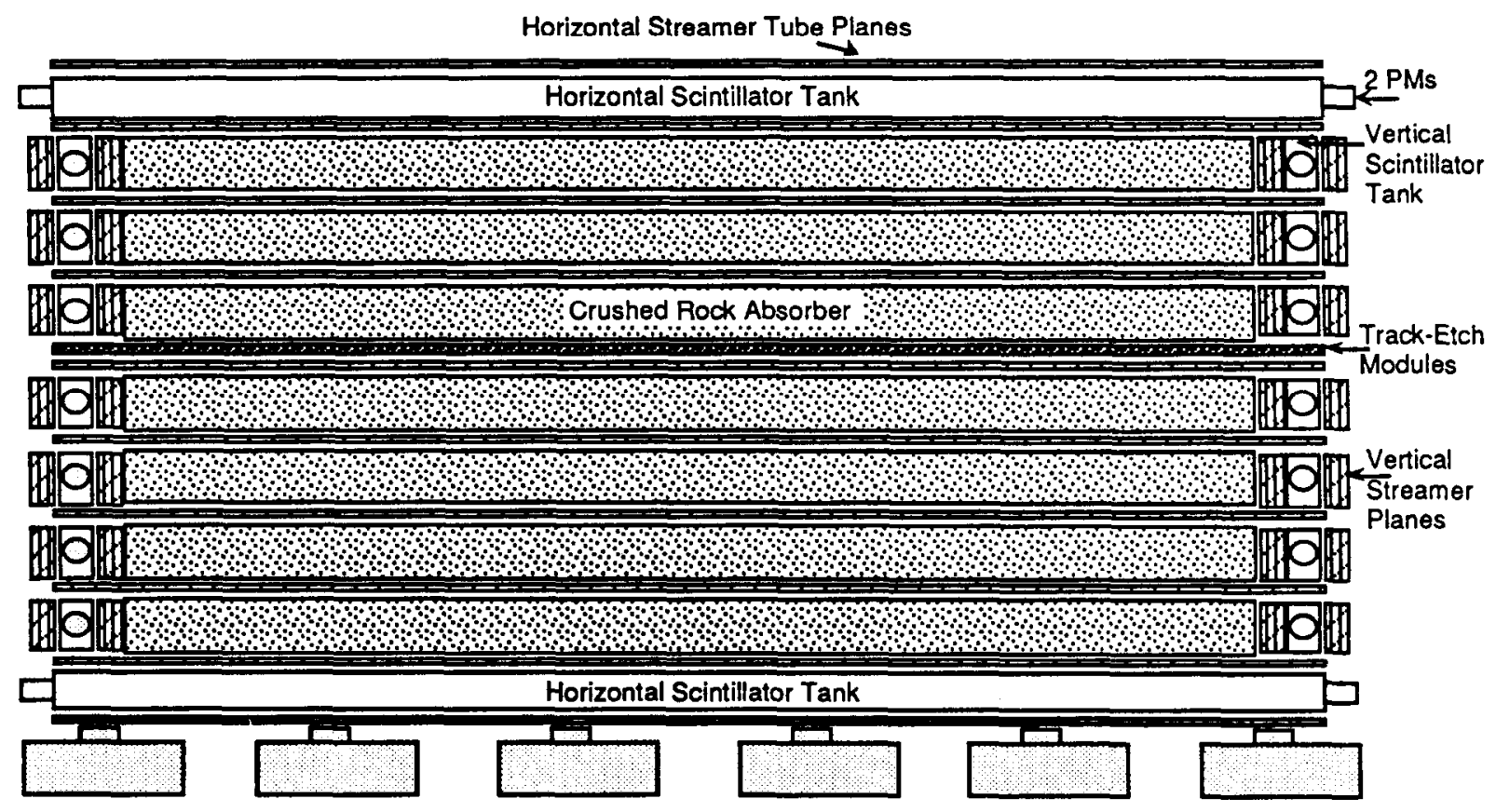

Fig. 1. A schematic drawing of the lower half of one supermodule of the MACRO experiment. 
core, the electron degeneracy pressure drops and the core collapses to a neutron star of nuclear density $10^{14} \mathrm{~g} / \mathrm{cm}^{3}$ and radius $\sim 10 \mathrm{~km}$. The compression of the stellar core to neutron star density releases a large amount of gravitational energy $\left(0.1-0.2 M_{\odot}\right)$, with most of this energy being emitted in the form of neutrinos. The collapse heats the core to a temperature of $10^{11} \mathrm{~K}$. Neutrino pairs of all flavors then radiate this energy and cool the core over an interval of several seconds. In the results from KAMIOKANDE II for SN1987A, eight out of the eleven events observed occurred in a $2 \mathrm{~s}$ interval [1]. The average energy of the $\bar{v}_{\mathrm{e}}$ produced is $E_{v}=10$ $\mathrm{MeV}$. The total energy released by core cooling is $\sim 3 \times 10^{53}$ ergs.

To characterize the expected neutrino flux $\Phi\left(E_{\nu}\right)$ we adopt a simplified constant temperature thermal neutrino spectrum of the FermiDirac type for the cooling stage:

$\Phi\left(E_{v}\right)=\frac{A_{0} E_{v}^{2}}{1+\exp \left(E_{v} / T\right)}$.

The values of the parameters have been taken from the statistical analysis of SN1987A data by Bludman and Schinder [6]. 'They give temperatures of $3.3 \mathrm{MeV}$ for $v_{\mathrm{e}}$ and $\bar{\nu}_{\mathrm{e}}$ and $6.6 \mathrm{MeV}$ for $\nu_{\mu, \tau}$ and $\bar{\nu}_{\mu, \tau}$.

The constants $A_{0}$ for the different neutrino types are related by $A_{0}\left(\nu_{\mu, \tau}\right)=A_{0}\left(v_{\mathrm{e}}\right) / 16$ with $A_{0}\left(v_{\mathrm{e}}\right)=5.21 \times 10^{55} \mathrm{MeV}^{-3}$. The total energy radiated through neutrinos of all types is $E=6 \times$ $5.862 A_{0} T^{4} \approx 3.4 \times 10^{53}$ ergs. The neutrino spectra obtained from the computer simulation of gravitational collapse by Mayle, Schramm and Wilson [7] are similar, but have somewhat larger high-energy tails and different ratios among the various neutrino fluxes.

\section{Neutrino detection in MACRO}

MACRO detects neutrinos through their interaction with liquid scintillator. The active volume of scintillator is divided into many individual counters. A schematic drawing of the completed lower half of MACRO is shown in fig. 1. A full description of the lower portion of the first supermodule has been presented elsewhere [8]. Here we shall examine in detail the capabilities of the detector for stellar collapse neutrino detection. Each supermodule will ultimately contain three layers of 16 horizontal scintillation counters, each with an active volume of $73.2 \times 19 \times 1120 \mathrm{~cm}^{3}$; each external face is covered with 14 counters, each with an active volume of $21.7 \times 46.2 \times 1107$ $\mathrm{cm}^{3}$. The liquid scintillator consists of $96.4 \% \mathrm{~min}-$ eral oil, $\sim 3.6 \%$ pseudocumene and small concentrations of scintillation fluors; it has a density of $0.86 \mathrm{~g} / \mathrm{cm}^{3}$ and a refractive index of 1.48 . The number densities of ${ }^{12} \mathrm{C}$ and ${ }^{1} \mathrm{H}$ nuclei are $3.7 \times$ $10^{22} \mathrm{~cm}^{-3}$ and $7.3 \times 10^{22} \mathrm{~cm}^{-3}$, respectively. The inside of all counters is lined with teflon $(n=1.33)$ for total internal reflection and there is an air gap above the liquid. Light is collected at each end of the counters by two PMTs and focusing mirrors, (one PMT at each end for vertical counters) which are assembled in end compartments filled with mineral oil and separated from the scintillator by clear PVC windows.

To calculate the response of the detector to neutrinos from a stellar collapse, we have used neutrino cross-sections on electrons and free protons from Bahcall [9]. Charged current (CC) and neutral current (NC) neutrino cross-sections on ${ }^{12} \mathrm{C}$ and in particular those for the superallowed (isovector, axial vector) transition to the $\left(1^{+}, T=\right.$ 1) $15.1 \mathrm{MeV}$ level have been calculated by several authors [10,11]. We have used the most recent calculation of this transition [12] based on data from inelastic scattering of neutrons on ${ }^{12} \mathrm{C}$ $[13,14]$. Recent measurements by the KARMEN group [15] confirm these calculations. The crosssections for reactions induced by neutrinos on protons and carbon nuclei are presented in fig. 2 . Table 1 lists the number of events that would be seen from a supernova at the Galactic center (8.5 $\mathrm{kpc}$ ) in a detector containing 600 tonnes of liquid scintillator and with a threshold for detected electron energy of $7 \mathrm{MeV}$.

The dominant reaction is $\bar{\nu}_{\mathrm{e}}+\mathrm{p} \rightarrow \mathrm{n}+\mathrm{e}^{+}$. The positron energy provides the primary trigger for the detector. This reaction is followed, after neutron moderation in the liquid scintillator, by neutron capture $\mathrm{n}+\mathrm{p} \rightarrow \gamma+\mathrm{d}$ with $E_{\gamma}=2.2 \mathrm{MeV}$. 


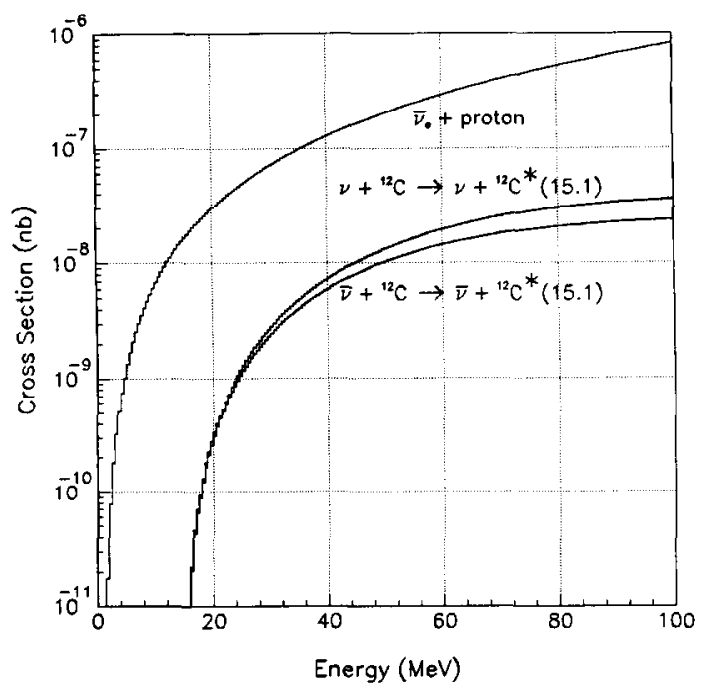

Fig. 2. Neutrino cross-sections on carbon nuclei and protons.

The average neutron moderation time is $10 \mu \mathrm{s}$, and the average capture time is $180 \mu \mathrm{s}$. The detection of the $2.2 \mathrm{MeV} \gamma$-rays is possible in MACRO because of the relatively large number of observed photoelectrons ( $\sim 20$ for an event at the longitudinal center of a scintillation counter) produced by a $1 \mathrm{MeV}$ Compton scattered electron.

\section{The gravitational collapse triggers and data acquisition systems}

Triggers for the selection of low-energy events induced by a supernova neutrino burst must be able to differentiate real events from background.

Table 1

Number of events from a stellar collapse at the Galactic center $(8.5 \mathrm{kpc})$ expected in MACRO; (600 tonnes of liquid scintillator) $E_{\mathrm{th}}^{\mathrm{e}}=7 \mathrm{MeV}$.

\begin{tabular}{llll}
\hline $\begin{array}{l}\text { Current } \\
\text { type }\end{array}$ & $\begin{array}{l}\text { Reaction } \\
\text { type }\end{array}$ & $\begin{array}{l}\text { Neutronization } \\
\text { burst }\end{array}$ & $\begin{array}{l}\text { Cooling } \\
\text { stage }\end{array}$ \\
\hline $\mathrm{CC}$ & $\bar{v}_{\mathrm{e}}+\mathrm{p} \rightarrow \mathrm{n}+\mathrm{e}^{+}$ & not applicable & 182 \\
$\mathrm{NC}$ & $v_{\mathrm{x}}+{ }^{12} \mathrm{C} \rightarrow v_{\mathrm{x}}$ & & \\
& $+{ }^{12} \mathrm{C} *(15 \mathrm{MeV})$ & 0 & 9 \\
$\mathrm{ES}$ & $v_{\mathrm{x}}+\mathrm{e} \rightarrow v_{\mathrm{x}}+\mathrm{e}$ & 0 & 2 \\
\hline
\end{tabular}

For the NC and elastic scattering (ES) reactions, $v_{\mathrm{x}}$ is equivalent to the sum of all $\nu$ and $\bar{v}$ flavors.
The problem of background rejection in the detection of stellar gravitational collapse is simplified by the pulsed character of the supernova. The event cluster generated by a neutrino burst from a supernova might be simulated by a statistical fluctuation above the average event rate. The dominant background components are cosmic rays and local radioactive decay.

Cosmic ray muons are observed as events with an average energy loss of about $40 \mathrm{MeV}$ and have a rate of $2 \mathrm{mHz}$ in a single scintillation counter. Cosmic rays can be effectively rejected by looking for coincidences among scintillation counters that are not adjacent, by identifying their large energy release and by position matching with tracks reconstructed in the streamer tube system. The rate of cosmic rays not eliminated by these signatures was less than $3 \mathrm{mHz}$ in the lower part of the first supermodule (45 tonnes), and should decrease substantially in the complete detector.

The natural background radioactivity originates primarily from the decay of radioactive isotopes present in the Gran Sasso rock, in the concrete used in the laboratory, and in the materials used within the experiment [16,17]. Gamma rays emitted by the radioactive isotopes of the ${ }^{238} \mathrm{U}$ and ${ }^{232} \mathrm{Th}$ decay chains or by ${ }^{40} \mathrm{~K}$ are detected in the scintillation counters after being partially degraded by preceeding interactions. Neutrons are a further source of $\gamma$ radiation, with energies extending up to nuclear binding energies. Neutrons can be captured both within the scintillator and in other materials interspersed within the detector. In a single scintillation counter, the background rates are $5 \mathrm{kHz}$ for energy deposition greater than $1 \mathrm{MeV}$ and $1 \mathrm{~Hz}$ for energy deposition greater than $4 \mathrm{MeV}$.

The large background due to radioactivity must be rejected with special trigger electronics. Due to light attenuation in the scintillation counters, a given energy deposited in a counter may correspond to PMT pulse amplitudes differing by up to a factor of six. The rejection of radioactivity over a given energy threshold requires a fast energy reconstruction independent of the event position within the counter. The trigger circuits for stellar gravitational collapse detection use a primary energy threshold in the range $E=5-10$ 
$\mathrm{MeV}$. To detect the $2.2 \mathrm{MeV}$ photon from n-capture on protons with reasonable efficiency, an energy threshold of $E \approx 1 \mathrm{MeV}$ (the secondary threshold) is required for an interval of at least several times the n-capture time in scintillator $(\tau \approx 180 \mu \mathrm{s})$ following the primary trigger.

The signals from the PMTs in the scintillation counters are distributed to the PHRASE (Pulse Height Recorder And Synchronous Encoder) and ERP (Energy Reconstruction Processor) systems. The PHRASE is a trigger specifically designed to detect neutrinos from gravitational collapse. The ERP system provides a general muon trigger from individual scintillation counters and also has good stellar gravitational collapse selection capabilities.

\subsection{The PHRASE system}

Each PHRASE module serves two scintillation counters. The main characteristics of the PHRASE circuit are:

(1) It provides an energy trigger (ET) signal when a threshold condition is satisfied on the energy released by an event in a single scintillation counter. To a good approximation, the energy threshold does not depend on the event position within a counter. This is accomplished in hardware by compensating for the light attenuation properties of the detector and by discriminating on analog signals which are proportional to the event energy.

(2) It digitizes and stores waveforms (100 $\mathrm{MHz}$ at eight bits effective resolution) from a particular counter when an ET has occurred *.

(3) It measures with an accuracy of $1.6 \mathrm{~ns}$ the time of each event relative to the experiment's atomic clock reference time. It also measures the time difference between the signals from the two counter ends with the same accuracy.

(4) When an ET is obtained from one counter, the energy thresholds for that counter and for the adjacent counters are lowered to a different programmable value for a presettable time, nominally $850 \mu \mathrm{s}$. PHRASE then records any events

\footnotetext{
* Waveform digitization is based on techniques developed for the circuit GATHER [18] at CERN.
}

exceeding the lowered threshold which occur during this interval to a maximum of 14 secondary events per channel for each primary event.

\subsection{The PHRASE energy trigger}

We choose a longitudinal coordinate axis $x$ with origin at the center of a scintillation counter of length $L$. The two counter ends are identified by $x=-\frac{1}{2} L$ for the "left" end and $x=+\frac{1}{2} L$ for the "right" end. Light attenuation in the detectors is approximately exponential with distance from the PMTs with attenuation length of roughly $\lambda=12 \mathrm{~m}$. The pulse amplitudes for the "left" and "right" PMTs can then be written

$$
A_{\mathrm{L}}(E, x) \propto E \mathrm{e}^{-x / \lambda} \text { and } A_{\mathrm{R}}(E, x) \propto E \mathrm{e}^{x / \lambda}
$$

for an event releasing a visible cncrgy $E$ at the longitudinal position $x$ inside the counter. The arrival time for light at the two PMTs can be written

$$
\begin{gathered}
t_{\mathrm{L}}=t_{\mathrm{MT}}+x / v \text { and } t_{\mathrm{R}}=t_{\mathrm{MT}}-x / v \\
\text { with } v \approx 0.2 \mathrm{~m} / \mathrm{ns},
\end{gathered}
$$

where $t_{\mathrm{MT}}$ is the measured mean time $t_{\mathrm{MT}}=\frac{1}{2}\left(t_{\mathrm{L}}\right.$ $+t_{\mathrm{R}}$ ). If an exponential tail pulse is electronically added to the peak amplitude signals, the pulse height can be written

$$
\begin{aligned}
& S_{\mathrm{L}}(E, x, t) \propto E \mathrm{e}^{-x / \lambda} \mathrm{e}^{-\left(t-t_{\mathrm{L}}\right) / \tau} \\
& \text { and } S_{\mathrm{R}}(E, x, t) \propto E \mathrm{e}^{x / \lambda} \mathrm{e}^{-\left(t-t_{\mathrm{R}}\right) / \tau} \\
& \text { for } t \geq t_{\mathrm{L}, \mathrm{R}} \text { and } 0 \text { otherwise. }
\end{aligned}
$$

If $\tau$ is chosen such that $\tau=\lambda / v$, the previous formulae simplify into

$$
\begin{aligned}
& S_{\mathrm{L}}(E, x, t) \propto E \mathrm{e}^{-\left(t-t_{\mathrm{MT}}\right) / \tau} \\
& \quad \text { and } S_{\mathrm{R}}(E, x, t) \propto E \mathrm{e}^{-\left(t-t_{\mathrm{MT}}\right) / \tau} \\
& \text { for } t \geq t_{\mathrm{L}, \mathrm{R}} \text { and } 0 \text { otherwise. }
\end{aligned}
$$

For times greater than the maximum of $t_{\mathrm{L}}$ and $t_{\mathrm{R}}$, the exponential tails are overlapping and their amplitudes, at a fixed delay $t^{*}$ after $t_{\mathrm{MT}}$, are functions of energy only. The exponential position non-uniformity is thus cancelled. In MACRO $\tau=60 \mathrm{~ns}$ and we choose $t^{*}=40 \mathrm{~ns}$, which always 
satisfies the condition $t=t_{\mathrm{MT}}+t^{*}>\max \left(t_{\mathrm{L}}, t_{\mathrm{R}}\right)$. The exponential tails are continuously compared with the primary energy threshold, and the two discriminator outputs go to a three-fold coincidence together with a $t_{\mathrm{MT}}$ signal. A trigger occurs when both tails are above the $7 \mathrm{MeV}$ threshold when the $t_{\mathrm{MT}}$ signals arrives. At this point the threshold is lowered to $1 \mathrm{MeV}$ for $850 \mu \mathrm{s}$ to detect secondary events.

\subsection{The PHRASE data acquisition system}

The data from two groups of three SMs are read out and are stored temporarily in two dedicated microcomputers. The data are then transferred to the main experiment computer and written into the MACRO run file together with the data from all other scintillation counter and streamer tube systems. The PHRASE stellar gravitational collapse data acquisition can be considered as a completely independent system right up to the general data storage on the main computer. The average dead time of the PHRASE configuration is very small $(\sim 0.004 \%$ for each SM) and as a result, the data acquisition system is not dead time limited, even in the case of an intense event burst from a supernova.

\subsection{The ERP system}

The ERP combines the functions of a muon trigger, stellar collapse trigger, and signal amplitude and timing readout of the scintillation counters in a single integrated system. Each ERP channel services one scintillation counter and has the following characteristics:

(1) A trigger based on the amount of energy deposited in the scintillation counter. The intent of this trigger is similar to the PHRASE trigger, but is obtained using a digital technique. In the ERP, a look up table (LUT) is loaded with the energy corresponding to all possible left/right signal pairs, to six bits of accuracy on the inputs. This technique allows a complete mapping of each counter's response as a function of event location. It also allows maximal flexibility because all aspects of the trigger are software programmable.
(2) An analog to digital converter/time to digital converter (ADC/TDC) system is incorporated into the ERP and is read out for each trigger. The signal from each counter end is input to two ADCs, with the gain of one ADC set a factor of $\mathbf{1 0}$ lower than the other. This allows the full dynamic range of the PMTs to be reliably recorded. The signal from each counter end is input to two TDC channels as well; their input discriminators have different thresholds to provide a means of identifying events in which the leading edge of the PMT pulse is distorted due to prepulsing or pileup.

(3) Each event read out by the ERP is stored in a local buffer, with a relative time accurate to 1 $\mu s$ stored with each event. Off line, the absolute time of each event is obtained with reference to the same atomic clock used by the PHRASE system.

\subsection{The ERP trigger}

The ERP trigger is based on reconstructing the energy deposition and position from the left and right signal pairs of a scintillation counter. This reconstruction requires that the response of the counter as a function of position and the relative gain of the electronics chain be known. The response at one end can be modeled very well by a function of the form

$$
A_{i}\left(x_{i}\right)=E\left(C_{1} / x_{i}^{2}+C_{2} \mathrm{e}^{-x_{i} / \lambda_{i}}+C_{3} \mathrm{e}^{-x_{i} / \lambda_{2}}\right),
$$

where $x_{i}$ is the distance from the $i$ th end ( $i=$ $\mathrm{L}, \mathrm{R})$ of the counter, $E$ is the deposited energy, and $C_{1}, C_{2}, C_{3}, \lambda_{1}$, and $\lambda_{2}$ are parameters we determine for each counter. This function accounts for the exponential behavior of the counters as well as the non-exponential behavior near the PMTs. Measurements of the pulse amplitudes at the "left" and "right" ends, $A_{\mathrm{L}}$ and $A_{\mathrm{R}}$, respectively, give both the energy deposition and position of the event. In the ERP trigger system $A_{\mathrm{L}}$ and $A_{\mathrm{R}}$ are digitized by 6-bit Flash ADCs (FADCs) employing a non-linear response of the form

$D=63 \frac{Q}{Q_{\max }-B\left(Q_{\max }-Q\right)}$, 
where $D$ is the digital output of the FADC, $Q$ is the input signal, and $B$ determines the degree of non-linearity. With $B=0.8$, approximately 1 bit is added to the effective dynamic range of the inputs. The two six-bit numbers from the FADCs are used to form a 12-bit address into a LUT. Each address contains two eight-bit words, one from the energy LUT and the other from the position LUT. The position word includes a six-bit position value and two trigger bits, corresponding to the high-level (12 MeV) trigger for muons and the low-level $(7 \mathrm{MeV})$ trigger for stellar collapse events.

\subsection{The ERP readout system}

In addition to providing a set of triggers for muon and stellar collapse events, the ERP functions as a complete readout system for the scintillation counters. The front end of the ERP system consists of a set of four sample and hold $(\mathrm{S} / \mathrm{H})$ circuits for each counter end. Two of these are used to hold pulse height information, while the other two hold timing information. The signals from the sample and hold circuits are used both in the readout of the system and as the input to the trigger described above. When a trigger occurs, these signals are read out through a system bus to a central readout supervisor which consists of a fast 12-bit ADC, zero suppression circuity, a local clock and a buffer memory. The least count of the $\mathrm{ADC}$ system is $1 \mathrm{pC} /$ count for the highgain $\mathrm{S} / \mathrm{H}$, and $10 \mathrm{pC} /$ count for the low-gain $\mathrm{S} / \mathrm{H}$. The dynamic range of the $\mathrm{ADC}$ system is $10 \mathrm{pC}$ to $40000 \mathrm{pC}$. The TDC readout has a least count of $160 \mathrm{ps}$.

The means of reading out the supervisor to the data acquisition (DAQ) system depends on the type of trigger generated. For a high-level muon trigger the ADC/TDC information is read out promptly. This allows the ERP muon data to be associated with the data from other readout electronics in a simple manner. The rate of low-level triggers, normally due to background radioactivity, is too high to allow a prompt readout. These events are locally buffered within the ERP supervisor in an $8 \mathrm{~K}$-word first in, first out (FIFO) buffer. When this FIFO is full, a readout request is issued to the DAQ system and the memory is dumped. In normal operation, the filling of a FIFO takes about 10 minutes, so that readout of the stellar collapse data sample represents a minimal overhead for the DAQ system.

\section{Calibration and testing}

Unfortunately, Galactic supernovæ do not occur frequently enough to calibrate and test the detector; alternate methods must be employed. The efficiency of the detector must be determined, and the energy scale must be accurately calibrated. Experimental conditions such as temperature, ventilation and power supply must be stabilized. Because electrical disturbances can cause false event clusters, the power lines are monitored continuously with a commercial power line disturbance monitor, and anomalies are automatically timed and recorded.

\subsection{Detection of neutron capture gamma rays}

$\mathrm{Am} / \mathrm{Be}$ is a widely used neutron emitter that has been used to verify that MACRO can detect neutron capture $\gamma$-rays and to measure the efficiency and spatial resolution of this detection. Sixty percent of the neutrons are associated with a cascade deexcitation $\gamma$-ray from the $4.4 \mathrm{MeV}$ ${ }^{12} C^{*}$ level, which we refer to as $\gamma_{4}$. The $\gamma_{4}$-ray provides the primary trigger which causes the thresholds to be lowered to allow detection of the neutron capture $\gamma$-ray.

The response of three of the MACRO scintillation counters to an $\mathrm{Am} / \mathrm{Be}$ source was studied in detail. The full energy spectrum observed is well reproduced by a Monte Carlo simulation (see fig. 3). The numbers of detected $\gamma$-rays from both the ${ }^{12} \mathrm{C}^{*}$ cascade $(4.4 \mathrm{MeV})$ and neutron capture $(2.2 \mathrm{MeV})$ agree within $10 \%$ with the values predicted [19]. Furthermore, the distribution of the time between the primary trigger and the secondary one $(1<E<4 \mathrm{MeV})$ is exponential with a characteristic time of $180 \mu \mathrm{s}$, as expected for neutron capture (fig. 4). With these data we estimate that the efficiency for detecting the neutron capture following a primary $\bar{\nu}_{\mathrm{e}}$ event 

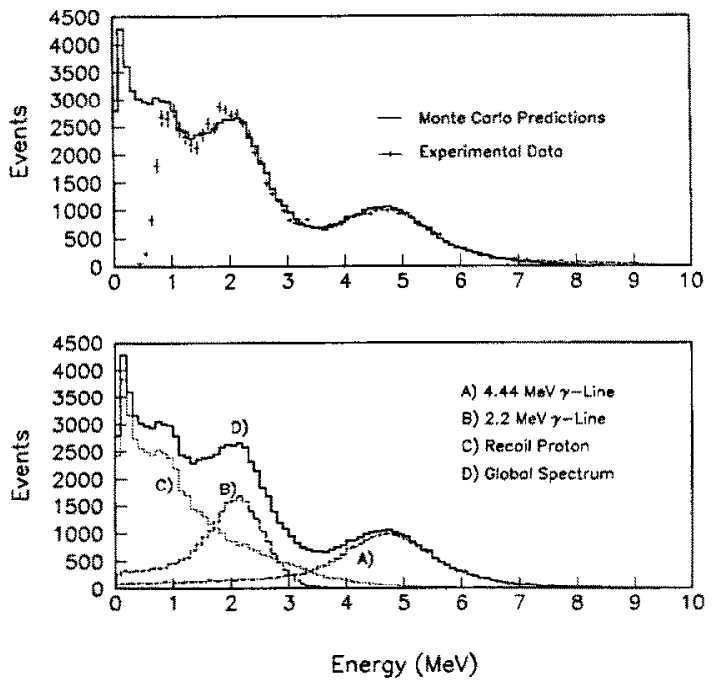

Fig. 3. The comparison between experimental data and Monte Carlo calculations for the spectrum induced by an $\mathrm{Am} / \mathrm{Be}$ source in a MACRO counter. The lower figures show Monte Carlo predictions for the separate components.

in the same counter (mimicked by a primary $\gamma_{4}$-ray from the $\mathrm{Am} / \mathrm{Be}$ source) is about $25 \%$ for a secondary energy threshold of $1 \mathrm{MeV}$.

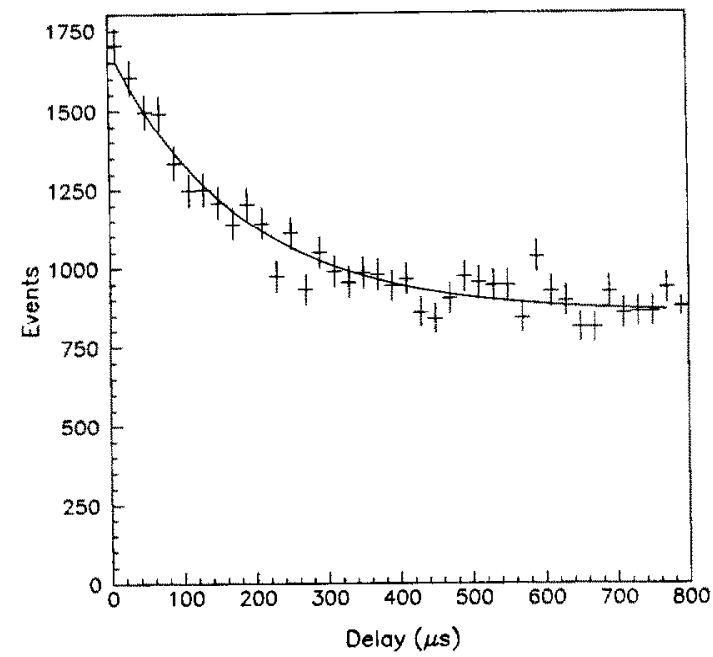

Fig. 4. Time distribution of secondary events occurring during the $800 \mu$ s time after the $\gamma_{4}$-primary with the Am/Be source. The curve is a fit to a constant background plus an exponential with a time constant of $180 \mu$ s. Position cuts have been applied.

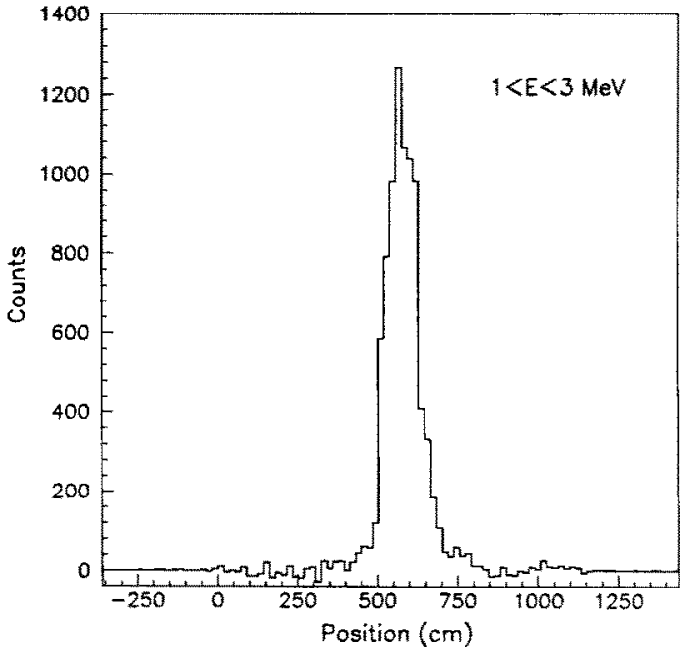

Fig. 5. Distribution of reconstructed positions for sccondary events along the counter. Natural radioactivity contributions were subtracted. The $\mathrm{Am} / \mathrm{Be}$ neutron source was uncollimated so the intrinsic position resolution is significantly better than indicated by the width of the peak.

The upper limit for the resolution in longitudinal position along the $12 \mathrm{~m}$ long scintillation counter for the n-capture $\gamma$-rays from the (uncollimated) $\mathrm{Am} / \mathrm{Be}$ source measurements is $\sigma_{z}<1$ $\mathrm{m}$, as shown in fig. 5 . This resolution is crucial, as a spatial correlation between the position of energy deposition by the primary reaction $\bar{\nu}_{\mathrm{e}}+\mathrm{p} \rightarrow$ $\mathrm{n}+\mathrm{e}^{+}\left(\sigma_{z} \approx 25 \mathrm{~cm}\right)$ and that of the secondary delayed n-absorption $\left(\sigma_{z} \approx 1 \mathrm{~m}\right)$ is a major tool for rejecting background from natural radioactivity when searching for stellar gravitational collapse $\bar{\nu}_{\mathrm{e}}$ events.

Systems for acquisition of stellar collapse data have problems due to the relatively high rate associated with low-energy thresholds. The raw rates are $0.3 \mathrm{~Hz}$ for primary events in the entire lower part of the first $\mathrm{SM}$ and $3 \mathrm{kHz}$ for $\mathrm{sec}$ ondary events in each counter. This corresponds to an average of fewer than 3 secondary events from natural radioactivity for every primary event within the $850 \mu \mathrm{s}$ window for the PHRASE. When position correlation, time correlation, and energy cuts are applied, signal/background is expected to be $\sim 2$. This capability of detecting neutron capture $\gamma$-rays with a secondary thresh- 


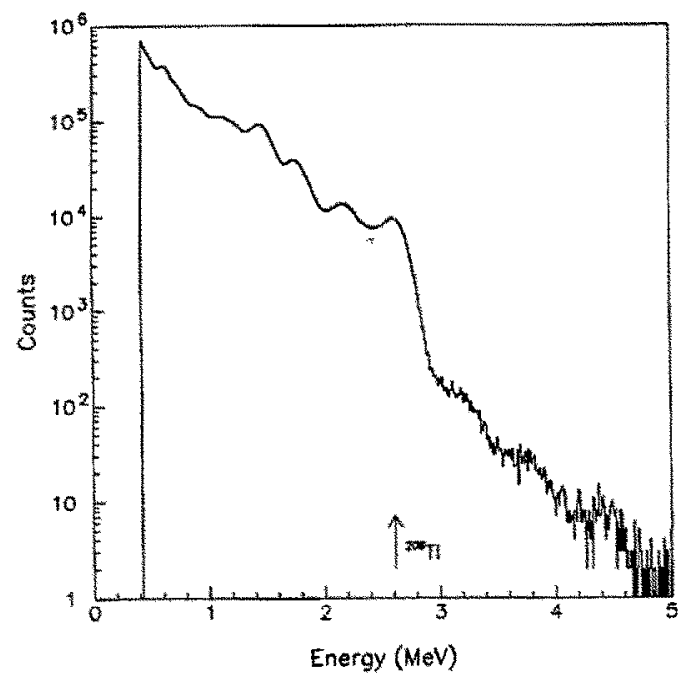

Fig. 6. The background radioactivity energy spectrum at the experimental site measured with a $\mathrm{Nal}$ detector.

old of $1 \mathrm{MeV}$ will be implemented for the entire detector.

\subsection{Calibration with cosmic ray muons}

Cosmic ray muons that pass through the detector provide a calibration point for the higher end of the energy scale. The trajectory of muons crossing the scintillation counters can be reconstructed from the information obtained by the scintillation counter system alone or, with higher accuracy, by the streamer tube tracking system. The average energy loss of vertical muons in the scintillation counters is $34 \mathrm{MeV}$. The muon event rate is $1 \mathrm{~m}^{-2} \mathrm{~h}^{-1}$, so several days are needed to calibrate the energy scale of each counter to approximately $5 \%$ accuracy. Muon events are also used to measure the response vs. position in each counter.

\subsection{Gran Sasso rock - the ${ }^{208} \mathrm{Tl} 2.614 \mathrm{MeV}$-ray line}

The Gran Sasso rock (and concrete) contains ${ }^{208} \mathrm{Tl}$ which emits a $2.614 \mathrm{MeV} \gamma$-ray that is used to determine another energy calibration point. When measured with a large $\mathrm{Nal}$ detector, the differential energy spectrum from background $y$ - rays in the Gran Sasso experimental halls appears as a rapidly decreasing continuum on which the ${ }^{208} \mathrm{TI}$ y-ray line is clearly visible (see fig. 6). Large liquid scintillation counters have much poorer energy resolution because of a lower number of photoelectrons per event and because of position dependent light attenuation effects. The position of an event is reconstructed using the difference in light transit time to the two ends of the counter, and the position compensated spectrum shows a distinct slope change at the ${ }^{208} \mathrm{Tl}$ line (see fig. 7). Using this line we are able to perform an energy calibration for each of the SMs within approximately $\pm 10 \%$ at a rate of roughly one SM per day without altering the data acquisition of the rest of the apparatus. This line thus provides a fast and valuable reference for setting primary and secondary thresholds for the stellar gravitational collapse data acquisition.

\subsection{Nonlinear interpolation of the MACRO energy scale}

Once the absolute energy reference points are fixed, the interpolation scale is calibrated. The non-linearity of the scintillation counter response is determined with a variable intensity pulsed ultraviolet (UV) light calibration utilizing an opti-

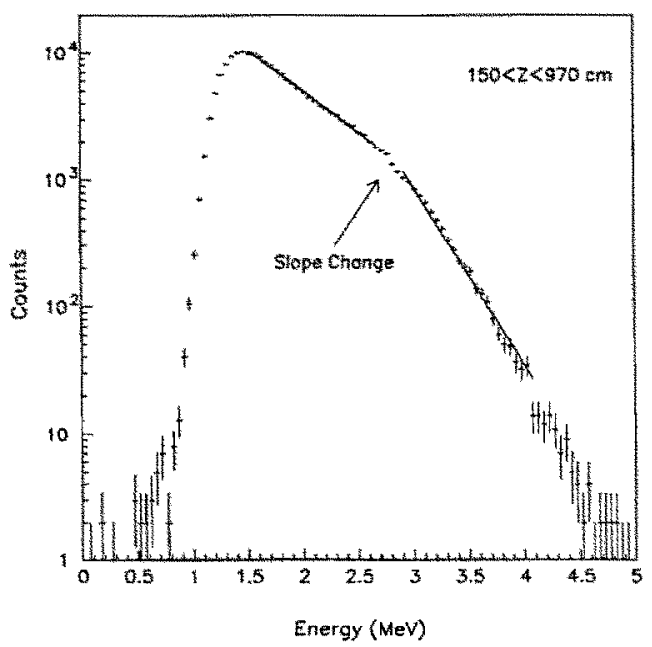

Fig. 7. The background radioactivity energy spectrum mea sured with a MACRO scintillation counter after compensation for position dependent effects. 
cal fiber driven by a UV laser at the center of each scintillation counter. The UV light stimulates isotropic visible light emission by the scintillator. The entire energy range of interest is calibrated to compensate for distortions due to PMTs, electronics, etc.

\subsection{The MACRO time standard}

The Gran Sasso Laboratory time standard is a commercial (ESAT RAD100) rubidium atomic clock. The $1 \mathrm{kHz}$ master clock signal is distributed to the various underground experiments by fiber optic cables. A slave clock in the underground laboratory produces an interpolating 10 $\mathrm{MHz}$ clock signal which is used by PHRASE and ERP. The accuracy of the Gran Sasso absolute time relative to UTC time is presently $100 \mu \mathrm{s}$. Better intercalibration and comparison with a reference clock are expected to improve this accuracy to $1 \mu \mathrm{s}$. The relative timing accuracy between scintillation counter events such as those expected for a $\bar{\nu}_{\mathrm{e}}$ burst is about 2 ns.

\section{Analysis methods used in the search for a stellar gravitational collapse $\bar{v}_{e}$ burst}

It is expected that in a core collapse supernova, $\bar{v}_{\mathrm{e}}$ burst detection should occur several hours before the detection of light emission. It is therefore very important both to identify a probable SN candidate by a fast online analysis and to rapidly verify its credibility by the use of the fully reconstructed event information. Prompt confirmation would allow the optical observation of the early stages of a new supernova by astronomical observatories.

The first indication of a possible $\bar{v}_{\mathrm{e}}$ burst, preliminary to a more careful event by event analysis, comes from a sudden increase in the counting rate of the entire scintillation counter system. An event filter and interpreter of the scintillation counting rate are provided as a lowpriority subprocess of the normal acquisition system which examines all events with an efficiency which is always $>99 \%$. Partial energy and position reconstruction are performed at this stage.

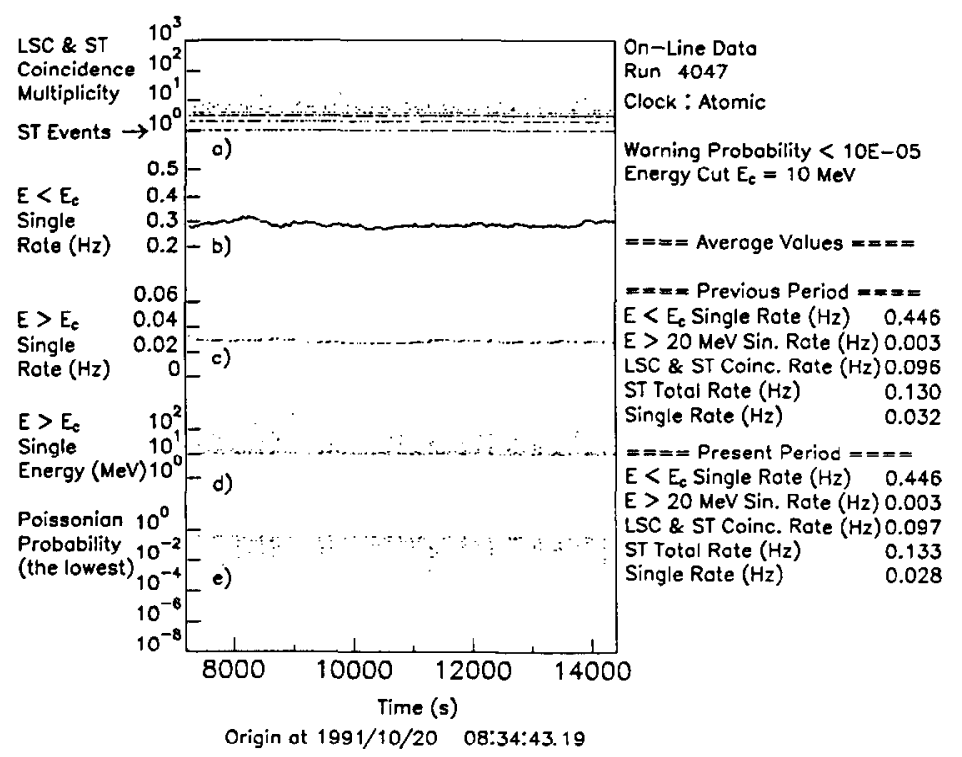

Fig. 8. The supernova online display which appears when the software detects a neutrino burst candidate. (a) presents the coincidence multiplicity for events in the scintillation counters (LSC) or streamer tubes (ST) over a 2 hour period; (b) and (c) show rates for singles having energies lower and greater than a chosen cut $\left(E_{\mathrm{c}}\right)$, respectively. The interpreter looks for event clusters in singles with $E>E_{\mathrm{c}}$. Each time a new event occurs, the event sequence is updated and the program computes, for several time intervals from $62.5 \mathrm{~ms}$ to $32 \mathrm{~s}$ in geometrical progression, the probability for the event clusters found in the event sequence. In (e), the lowest of these probabilities is presented. 
The events are divided into two categories:

(1) coincidences, consisting mostly of penetrating muons,

(a) events with two or more counters triggered within $160 \mathrm{~ns}$,

(b) events associated with a subsequent streamer tube system trigger within an appropriate delay;

(2) singles, which consist of all other events.

An important piece of information provided by this process is a running record of the singles counting rate as a function of time. This rate is computed by using the last 150 singles events. Also calculated at this point is information such as the number of scintillators involved in coincident events, the rate of such events, the rate of streamer tube triggers and the probability of particular event clusters. These data, collected over the previous 2 hours, are automatically displayed if the software detects a neutrino burst candidate (fig. 8).

Any abnormal event cluster whose probability is less than a preset level immediately generates an alert via computer nets and phone lines. This initiates a complete analysis by experts of the events in the cluster. A $\bar{v}_{\mathrm{e}}$ burst is identified as one that exceeds given probability cuts and has the qualitative characteristics expected for a genuine supernova. We are implementing a special early warning system to minimize the time required to notify other observers that a neutrino burst has been detected.

\section{Results of the stellar gravitational collapse search}

MACRO has been actively searching for a $\bar{v}_{e}$ burst from stellar gravitational collapse since October, 1989. During most of this period, only the horizontal layers of scintillator in the first supermodule ( $\sim 45$ tonnes of liquid scintillator) were collecting data. Since the spring of 1991 two additional supermodules were put into operation but remained active for only a limited time because of the ongoing construction of other parts of the experiment. The total time interval of the search included in this analysis was 29 months. A

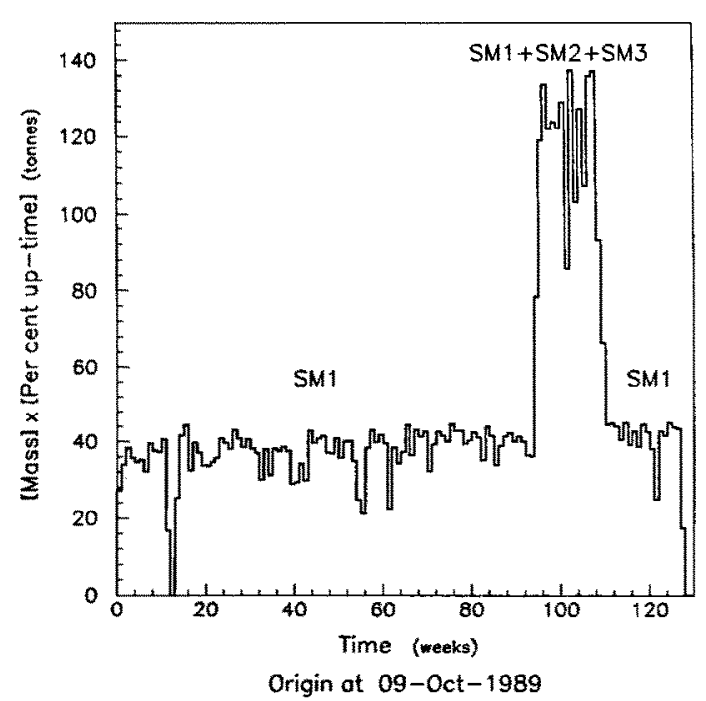

Fig. 9. Active scintillation mass vs, time. The mass has been multiplied by a factor to correct for live-time fraction.

plot of active scintillator mass versus time is shown in fig. 9. A factor has been included to account for down time attributable to maintenance, calibrations and power failures. The down time has now been reduced to about $5 \%$ and will eventually be negligible.

A search for event clusters was performed by applying software energy cuts $(E=7,8,9,10$ and $12 \mathrm{MeV}$ ) and burst durations (from $62.5 \mathrm{~ms}$ to 32 $s$ in geometrical progression, each step a factor of 2) to the filtered data sample. Since sensitivity to $\bar{\nu}_{\mathrm{e}}$ bursts depends on the integral background radioactivity, we present data here for an energy cut at $10 \mathrm{MeV}$. This energy cut results in a good signal to noise ratio for 45 tonnes of liquid scintillator and a Galactic supernova. As larger masses of liquid scintillator become active and the signal to noise ratio is improved, the software energy threshold can be reduced. Figure 10 shows the integral event rate over threshold vs. the energy threshold for 45 tonnes of scintillator. Figures 11 and 12 show, for the full period, the expected and measured numbers of clusters vs. their multiplicity for time intervals of 2 and $5 \mathrm{~s}$. The multiplicity is defined as the number of primary events observed within the selected time interval. There is agreement between measured and expected dis- 


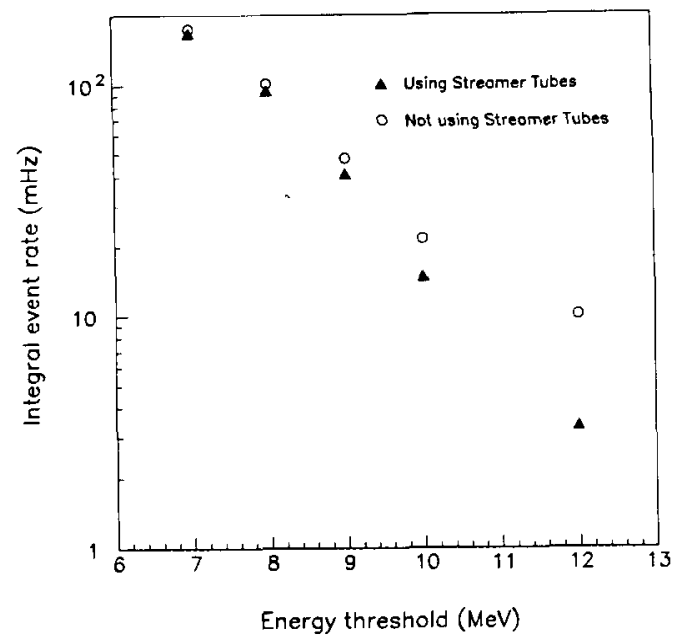

Fig. 10. Integral event rate over threshold vs. energy threshold. The data represented by the open circles are from the scintillation counters alone, while the filled triangles represent data where the streamer tubes have been used to remove penetrating cosmic ray muons.

tributions. Figure 13 shows the number of clusters vs. the burst duration for several cluster multiplicities. The agreement with Poisson expectations is good.

The sensitivity of SM1 to stellar collapse can be seen more clearly in fig. 14. This plot shows the allowable background radioactivity rate de-

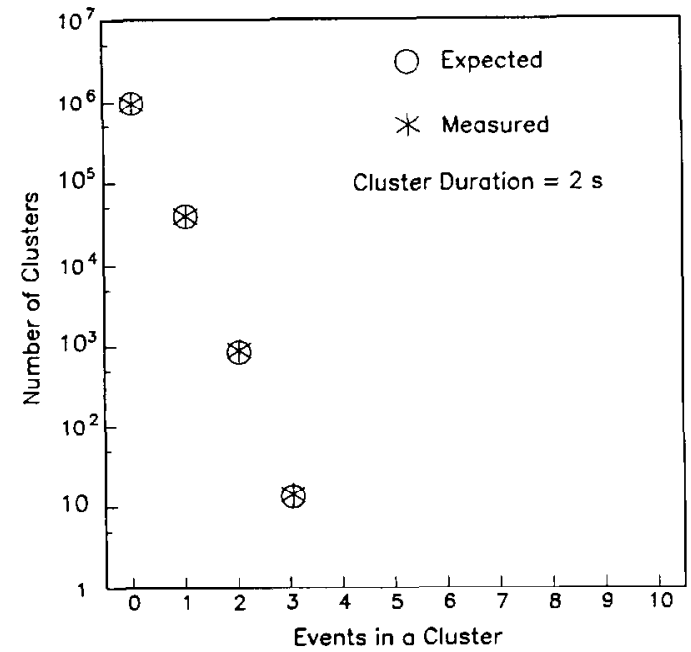

Fig. 11. Number of event clusters vs. cluster multiplicity for a time interval of $2 \mathrm{~s}$.

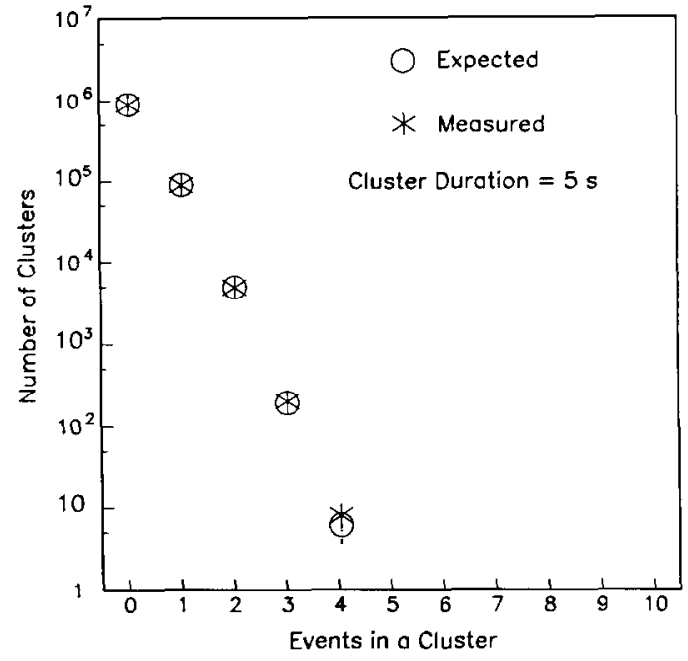

Fig. 12. Number of event clusters vs. cluster multiplicity for a time interval of $5 \mathrm{~s}$.

fined by specified probabilities $\left(10^{-1}, 10^{-3}\right.$ and $10^{-5}$ ) of having a false SN detection in ten years for a specified SN burst size (number of primary events) and duration ( $2 \mathrm{~s})$. The average background rate for SM1 is $\sim 15 \mathrm{mHz}$ for an energy cut of $10 \mathrm{MeV}$. Using this energy cut, the expected number of detected primary events is 11 for a $\mathrm{SN}$ at the Galactic center, assumed to be at $8.5 \mathrm{kpc}$ [20].

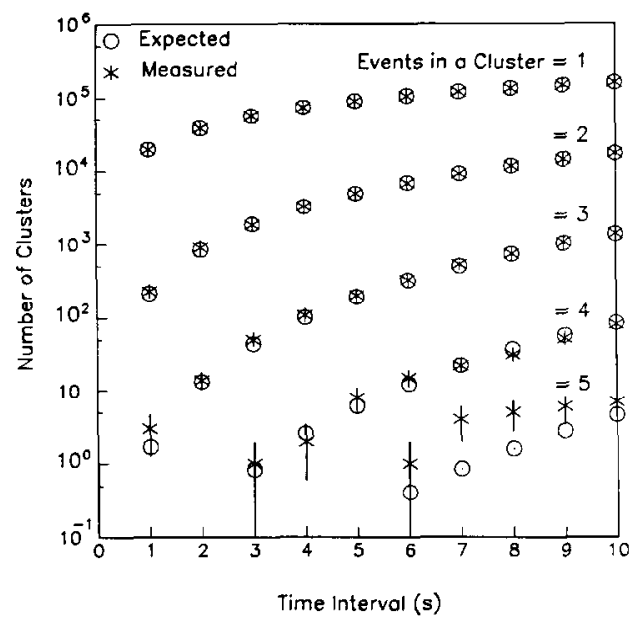

Fig. 13. Number of background event clusters vs. time interval for multiplicities 1, 2, 3, 4 and 5. Data (stars) are compared with expectations (circles). 


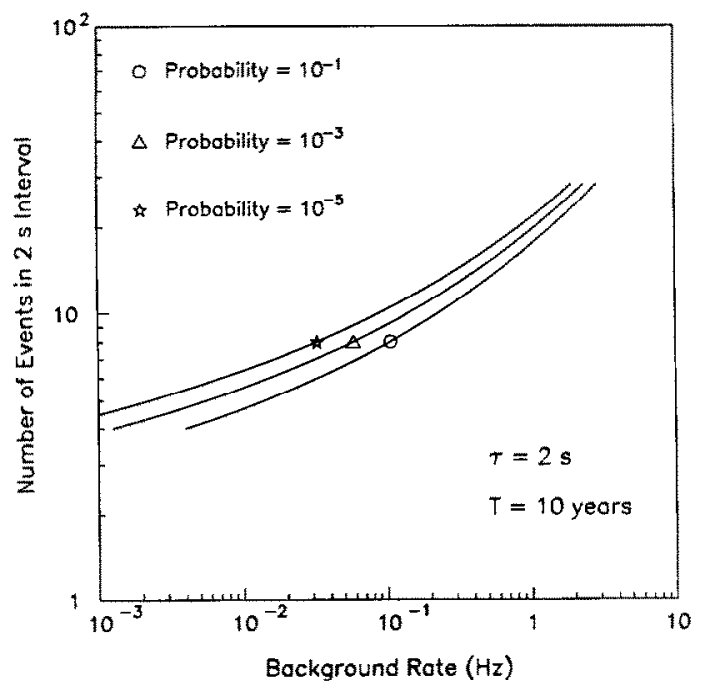

Fig. 14. Probabilities for observing a given number of events in a 2 second interval for a running time of 10 years vs. background rate.

A supernova search involves a tradeoff between signal and noise. Increasing the time interval beyond a few scconds will capture most of the signal at the expense of increased background. Reducing the time interval will minimize the background, but the signal will be reduced by an efficiency factor. For SN1987A as seen by the KAMIOKANDE-II detector [1], this efficiency would be $73 \%$ ( 8 out of 11 events) for a two-second interval. For a similar cvent occurring at the

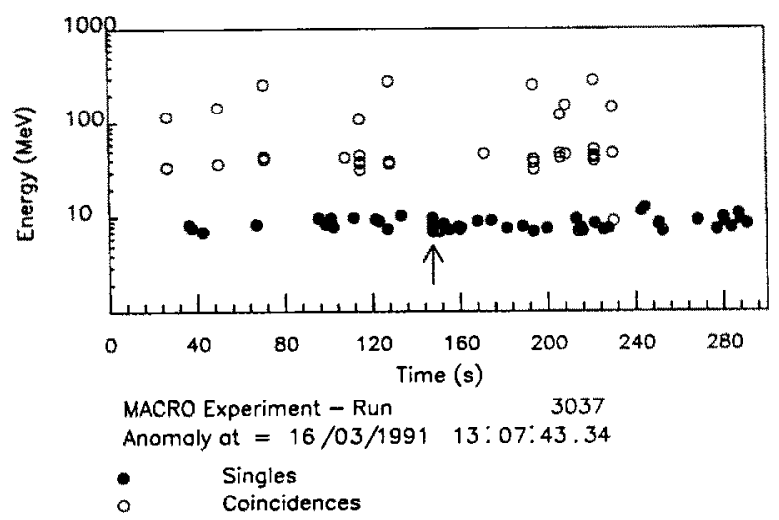

Fig. 15. Time sequence and energies for events in one particular cluster. The arrow indicates the cluster which contains 5 events very close to threshold in $500 \mathrm{~ms}$.
Galactic center, we would expect to see 8 events in $2 \mathrm{~s}$ with 45 tonnes of liquid scintillator using a primary trigger threshold of $10 \mathrm{MeV}$. Once a supernova candidate is found, the time interval can be expanded for subsequent analysis.

Once a candidate event cluster is selected, it is analyzed further to determine if the time sequence and energies of the events in the cluster are consistent with the signature of a $\bar{\nu}_{\mathrm{e}}$ burst. An example ( $E_{\mathrm{th}}=7 \mathrm{MeV}$ threshold, 5 events in 500 $\mathrm{ms}$ ) is presented in fig. 15. An arrow marks the event cluster. As one would expect for a background candidate, most of the events have energies close to the threshold. Events corresponding to coincidences with the streamer tube system or triggers which are identified as cosmic ray muons can also be removed.

\section{Discussion and conclusions}

The MACRO detector has achieved operation as a stellar collapse neutrino observatory. During the 2.5 year period beginning in October 1989 we operated the experiment in modes intended to calibrate the detector and verify its capabilities while maintaining a watch for neutrino bursts. Two complementary and redundant trigger and data acquisition systems have been developed and implemented that will allow us to take advantage of some of the unique signatures that a gravitational collapse event would produce in liquid scintillator. In particular, we have studied our ability to observe the delayed neutron capture $\gamma$-rays following the initial burst of positrons that would signal such an event. Our operational sensitivity now extends to most of the stars in our Galaxy and will cover the entire Galaxy when MACRO becomes fully operational.

During the two year period of this search, we did not see any indication of a neutrino burst giving more than four events in a two second period. With an efficiency of $73 \%$ and a threshold energy of $10 \mathrm{MeV}$, MACRO would have observed five events within a two second interval for an event similar to SN1987A occurring at a distance of $11 \mathrm{kpc}$. Although such an event could not be accepted as a supernova on the basis of 
probability arguments alone, an offline analysis would enable us to distinguish a genuine collapse.

To put these results in perspective, it is useful to determine the fraction of stars in our Galaxy sampled by a search sensitive to a stellar collapse within a given distance from our solar system. Based on the results of Bahcall and Piran [21], approximately $62 \%$ of the stars are within $11 \mathrm{kpc}$. In terms of a potential gravitational collapse sample, our 25 month search of $62 \%$ of the Galaxy is equivalent to a 15.5 month search of the full Galaxy.

In the coming years MACRO will increase its active scintillator mass. A program to minimize dead time is well underway, and we will continue to monitor event clusters for potential supernova candidates. The frequency of core collapse supernovæ (type Ib and II) for our Galaxy has been recently estimated [22] to be $7.3 h^{2}$ per century where $h$ is the Hubble parameter in units of 100 $\mathrm{km} \mathrm{s}^{-1} \mathrm{Mpc}^{-1}$. Assuming a ten year lifetime, the probability that MACRO will observe such a supernova is between $17 \%$ and $52 \%$ for $h$ between 0.5 and 1 . We expect that a Galactic supernova will produce enough neutrino events in the full detector to allow us to examine the time development of the neutrinosphere in the few seconds following core collapse.

\section{Acknowledgements}

We gratefully acknowledge the continuous cooperation and support of the director and staff of the Gran Sasso National Laboratory. This work was supported in part by the US Department of
Energy, the US National Science Foundation, and the Italian National Institute for Nuclear Physics (INFN).

\section{References}

[1] K.S. Hirata et al., Phys. Rev. Lett. 58 (1987) 1490.

[2] M. Aglietta et al., Europhys. Lett. 3 (1987) 1315. E.N. Alexeyev et al., Phys. Lett. B 205 (1988) 209.

[3] M. Calicchio et al. (The MACRO Collaboration), Nucl. Instrum. and Methods A 264, (1988) 18.

[4] M.S. Turner et al., Phys. Kev. D 26 (1982) 1296.

[5] W.D. Arnett et al., Ann. Rev. Astron. Astrophys. 27 (1989) 629.

[6] S.A. Bludman and P.J. Schinder, Astrophys. J. 326 (1988) 265.

[7] R. Mayle et al., Astrophys. J 318 (1987) 288.

[8] S.P. Ahlen et al. (The MACRO Collaboration), Nucl. Instrum. Methods, submitted.

[9] J.N. Bahcall, Neutrino Astrophysics (Cambridge Univ. Press, Cambridge, 1989).

[10] S.L. Mintz, Phys. Rev. Lett. C 25 (1982) 1671.

[11] T.W. Donnelly et al., Phys. Rep. 50 (1979) 1.

[12] M. Fukugita et al., Phys. Lett. B 212 (1988) 139.

[13] B.T. Chertok et al., Phys. Rev. C 8 (1973) 23.

[14] U. Deutschmann et al., Nucl. Phys. A 411 (1983) 337.

[15] B. Bodman et al, (The KARMEN Collaboration), Phys. Lett. B 167 (1991) 321.

[16] P. Belli et al., Nuovo Cimento A 101 (1989) 959.

[17] A. Alessandrello et al., Nuovo Cimento A 103 (1990) 1617.

[18] F. Bourgeois, Nucl. Instrum. Methods 219 (1984) 153.

[19] A. Baldini et al., Nucl. Instrum. Methods A 305 (1991) 475 .

[20] F.J. Kerr and D. Lynden-Bell, Mon. Not. R. Astron. Soc. 221 (1986) 1023.

[21] J.N. Bahcall and T. Piran, Astrophys. J. Lett. 267 (1983) L77.

[22] S. van den Bergh and G. Tammann, Ann. Rev. Astron. Astrophys. 29 (1991) 363. 